\title{
BMJ Open Conceptualising post-stroke fatigue: a cross-sectional survey of UK-based physiotherapists and occupational therapists
}

\author{
Karen Thomas (1) , Clarissa Hjalmarsson (D) , Ricky Mullis, Jonathan Mant
}

To cite: Thomas $\mathrm{K}$, Hjalmarsson C, Mullis R, et al. Conceptualising post-stroke fatigue: a cross-sectional survey of UK-based physiotherapists and occupational therapists. BMJ Open 2019;9:e033066. doi:10.1136/ bmjopen-2019-033066

- Prepublication history and additional material for this paper are available online. To view these files, please visit the journal online (http://dx.doi. org/10.1136/bmjopen-2019033066).

Received 19 July 2019 Revised 12 November 2019 Accepted 18 November 2019

D) Check for updates

(C) Author(s) (or their employer(s)) 2019. Re-use permitted under CC BY-NC. No commercial re-use. See rights and permissions. Published by BMJ.

Department of Public Health and Primary Care, University of Cambridge, Cambridge, UK

Correspondence to

Karen Thomas;

kt439@medschl.cam.ac.uk

\section{ABSTRACT}

With survival after stroke improving, more people are discharged into the community with multiple and persistent deficits. Fatigue is a common unmet need for stroke survivors, but there are no evidence-based guidelines for its assessment and management. This study explored how UK-based therapists conceptualise poststroke fatigue (PSF) in current practice.

Objective To describe current understanding of PSF among physiotherapists (PT) and occupational therapists (0T).

Design A cross-sectional online survey using Qualtrics software (a survey creation and analysis programme) was sent to therapists working with stroke survivors in 2019. Responses to the open ended question, 'How would you describe PSF if approached by another healthcare professional?' were analysed thematically by two independent researchers.

Participants 137 survey respondents (71 PT and 66 OT) from a range of clinical settings ( 25 acute care, 24 sub-acute rehabilitation care, 3 primary care and 85 community care) with 7 months-36 years of experience working with stroke survivors completed the survey. Results Respondents stated that PSF should be regarded as an important medical condition because it is common and can be associated with severe symptoms. Symptoms were perceived to be highly variable and the syndrome was difficult to define objectively. It was felt to have both physical and cognitive components. A variety of different opinions were expressed with regard to causation, conceptualisation and best management.

Conclusion Therapists working with stroke survivors conceptualise and manage PSF in different ways. Clinical practice is hampered by a lack of a widely adopted definition, and a small evidence base. Research into causes and management of PSF is a priority.

\section{INTRODUCTION}

Post-stroke fatigue (PSF) is a common symptom leading to unmet need for stroke survivors. ${ }^{12}$ It is a debilitating condition that adversely affects quality of life, social participation, return to work and mortality. ${ }^{3-5}$ Despite this, PSF lacks an agreed definition, gold-standard outcome measure or an evidence-based therapeutic option. ${ }^{6-8}$ Recent
Strengths and limitations of this study

- Survey design enables an efficient and nationally representative view of current clinical practice.

- Survey design removes the influence an interviewer may have on responses.

- The large number of respondents for a qualitative study means that it is unlikely that we failed to capture important themes.

- Targeting only a specialist sub-population of therapists who routinely deal with post-stroke fatigue coupled with the $8 \%$ response rate may mean our findings were unrepresentative of broader clinical practice.

- The open nature of the survey question may have resulted in different interpretations of the study question.

qualitative work contextualising PSF from a stroke survivor and caregiver perspective highlighted that acknowledgement of PSF provided legitimacy, but healthcare professionals did not approach PSF in a consistent way. ${ }^{9}$ In order to understand why this is, it is important to gain insight into the perspective of healthcare professionals on their understanding and management of the condition. ${ }^{3}$ Knowledge of both patient and clinician perspective can inform current fatigue management pathways.

Previous work has sought to classify PSF into biological and psychological dimensions, and to identify primary and secondary aetiology. ${ }^{10}{ }^{11}$ In 1891, Mosso delineated two clear aspects of fatigue, drawing a distinction between the diminution of muscular force and the sensation of fatigue. He split this into physical fatigue, which is readily measurable, and a more elusive psychological element. ${ }^{12}$ This classification continues to be used to explain a range of possible causative processes for fatigue. ${ }^{13}$ Ongoing research in the field aims to understand the aetiology 
and mechanisms of PSF in greater depth, as definitive conclusions have not yet been reached. ${ }^{14-16}$ Lynch and colleagues proposed the first case definition of PSF in 2007 and demonstrated its reliability and validity on stroke inpatients. ${ }^{17}$

This is the first study to investigate how therapists regard PSF in current practice by posing the question 'How would you describe PSF to another healthcare professional?'

\section{METHOD}

\section{Patient and public involvement}

Researcher KT visited a Cambridgeshire-based stroke group to discuss their understanding and experience of PSF. Possible topics were informed by the current literature, including guidelines and recommendation documents. ${ }^{18} 19$ The group gave feedback that improving understanding and management of PSF should be a priority.

\section{Question development}

A 10 question survey on the definition, assessment and management of PSF in clinical practice was developed by a multidisciplinary group (physiotherapists, occupational therapists and a clinical psychologist working in Cambridgeshire community teams). This study focuses on a single question relating to understanding of PSF which was created by lead author KT and then discussed and refined within the development group. The questions were piloted by three PT and one OT, as a result of which alterations were made. The open ended question was revised following discussion at the questionnaire development group to specifically target healthcare professionals.

\section{Participants}

PT who were members of the Association of Chartered Physiotherapists in Neurology (ACPIN) and OT who were members of Royal College of Occupational Therapy (RCOT) Specialist Section-Neurological Practice (RCOT-NSS) and who had registered an interest in participating in related research were invited to participate. To be eligible, participants were also required to have current registration as a healthcare professional, and to have worked with stroke survivors while in clinical practice within the UK.

\section{Design}

A cross-sectional survey (online supplementary file) was created and answered electronically using Qualtrics software, an online survey creation and analysis site licensed to the University of Cambridge (https://eu.qualitrics. com).

\section{Questionnaire distribution}

The research lead from each national organisation (ACPIN and RCOT-NSS) distributed an initial invitation email to members who had expressed an interest in participating in stroke-related research. Within the email was a participant invitation including a link to the Qualtrics platform which provided a participant information sheet and a consent form. Respondents entered their answers directly on to the Qualtrics online platform. After 2 weeks, a reminder email was sent to individuals who had not completed the survey. The survey remained live for 1 month following the reminder.

\section{Data analysis}

Submitted survey responses were collated on the Qualtrics platform (https://www.qualtrics.com/blog/citingqualtrics/) and downloaded using https secure protocol to the Secure Data Hosting Service at the University of Cambridge. These data were then anonymised and loaded into Microsoft Excel. Responses to question 5 'How would you describe PSF if approached by another healthcare professional?' were analysed thematically by two independent researchers KT and $\mathrm{CH}$. KT and $\mathrm{CH}$ first familiarised themselves with the data set. Both researchers coded $5 \%$ of the data to ensure a consistent coding strategy, then $40 \%$ of posts were randomly selected and coded by researcher $\mathrm{CH}$ while the remaining $60 \%$ were coded by KT. After all data had been coded, both researchers met and discussed the allocated codes, re-coding the data until unanimous agreement was reached. Codes were then grouped into sub-themes which were organised to create a thematic model. All responses were checked by both researchers to ensure all data fitted into the model. Descriptive statistics were used to analyse respondent characteristics and decipher frequencies within the data set.

We used the Standards for Reporting Qualitative Research (SRQR) checklist when writing our report. ${ }^{20}$

\section{RESULTS}

\section{Participant characteristics}

700 OT and 800 PT were sent a study invitation email of whom 137 completed the survey. Responses varied considerably in length from 2 to 148 words, with a median of 49 words. OT and PT responded in similar numbers (66 OT and 71 PT). Respondents had experience working in a variety of settings with stroke survivors: acute care (25 respondents), sub-acute rehabilitation care (24), community care (85) and primary care (3). The duration of specialism individuals had in stroke ranged from 7 months to 36 years (table 1 ).

\section{Themes}

Overview

The survey question, 'How would you describe PSF if approached by another healthcare professional?' generated a range of responses which were grouped into three broad themes shown below in figure 1: the importance of PSF; its legitimacy as a medical condition; the variety of ways in which healthcare professionals approach it. 
Table 1 Respondent characteristics by profession, clinical setting and duration of specialism

\begin{tabular}{ll}
\hline Participant respondents & Number \\
\hline Profession & \\
\hline Occupational therapist & 66 \\
Physiotherapist & 71 \\
\hline Clinical setting & \\
\hline Acute & 25 \\
Sub-acute & 24 \\
\hline Community & 85 \\
\hline Primary care & 3 \\
\hline Duration of specialism & \\
\hline <1 year & 4 \\
1-5 years & 29 \\
\hline $6-10$ years & 41 \\
\hline $11-15$ years & 17 \\
\hline 16-20 years & 19 \\
\hline 21+ years & 27 \\
\hline
\end{tabular}

Important: challenging, common and variable nature

A common response was that PSF was debilitating and deeply pervasive among stroke survivors. Respondents described PSF by using the descriptor 'overwhelming', and various other words to that effect. The term 'debilitating', and descriptors such as 'all-consuming', 'extreme' and 'tiredness like no other' were typical.

\section{Challenging}

Descriptive language was a marked feature of the responses, as therapists responded creatively to the

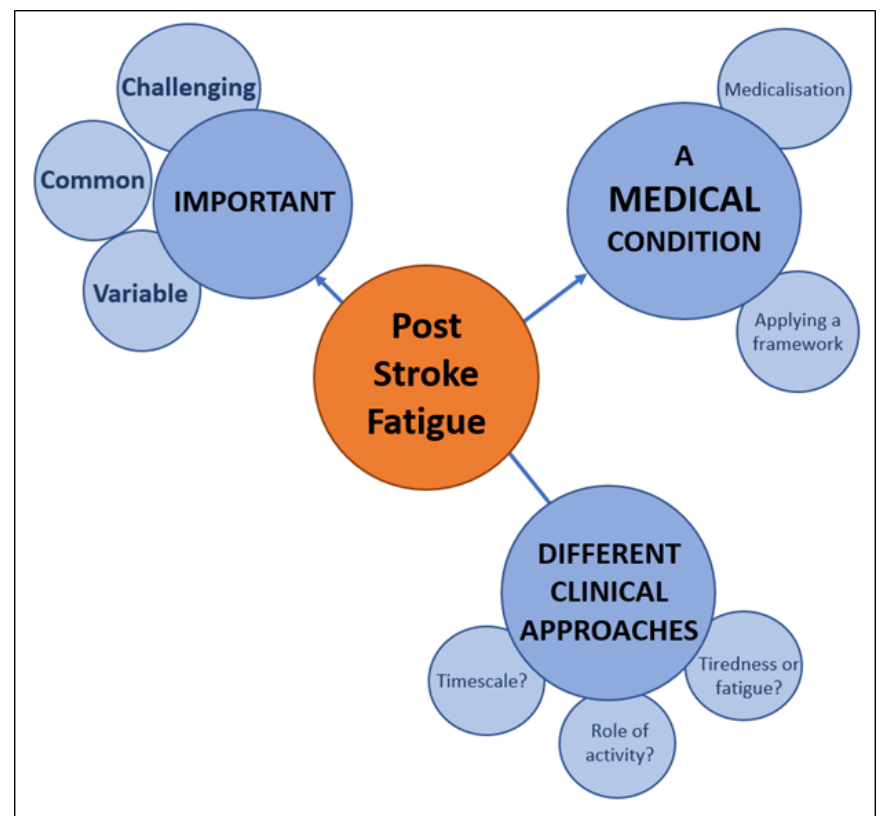

Figure 1 A visual representation of key themes arising in answer to the question 'How would you describe post-stroke fatigue to another healthcare professional?' challenge of explaining the difficulties of PSF to colleagues. Further, respondents highlighted the challenging nature of PSF and the effects this had on their patients. Answers stated, 'patients (sic) often feel like they are 'thinking through mud' (OT09) and 'pulling heavy boots on' (OT32). Others emphasised the distressing nature of PSF, describing it as 'hitting a brick wall' (OT62), and 'like your brain had been in a washing machine on a spin cycle' (OT47). Medicalised metaphors were also used, one of which likened the fatigue to 'sensory overload' (PT04).

Extended analogies were employed to convey the experience of living with PSF. One response suggested, 'I sometimes describe it as a bruise on the brain, and if you imagine a bruise on your ankle, you can see it's(sic) sore and stiff and you can't walk much before needing to rest' (OT27). One respondent directly referred to using 'the spoons analogy' (OT58). Coined by Christine Miserandino in 2003 to describe her experience of lupus, 'Spoon Theory' explains fatigue associated with chronic medical conditions by referring to only having a limited number of 'spoons' of energy that the patient can 'spend' during the day (https://butyoudontlooksick.com/articles/written-by-christine/the-spoon-theory/). In a similar manner, another reported, 'I describe the brain/body has having a 'battery' just like a car ... Post stroke the battery can be less full to start with, can be used up more quickly than usual and can take longer to recharge and not always fully' (PT08).

\section{Common}

Pervasiveness was highlighted as a key issue; respondents typically used the terms 'common', 'very common' and 'extremely common' to describe PSF, often to start their response. Efforts to quantify this frequency were mixed; one answer asserted that PSF 'affects nearly all stroke survivors' (OT55), and another that '(PSF) affects a large number of patients, approximately $25 \%$ (are) experiencing severe fatigue with a further $25 \%$ experiencing moderate fatigue' (PT47). Another felt that, 'Following stroke approx. $75 \%$ of patients experience fatigue as a symptom’ (OT25).

\section{Variable}

Respondents emphasised the complex and variable nature of PSF as a condition, describing it as having several components or affecting multiple aspects of a patients' life. Respondents attributed PSF to 'a mix of physical and emotional factors' (OT03), or provided more detailed causative processes, such as 'hormones, neurotransmitters and cognitive load ... exacerbated by secondary factors, such as diet, sleep and medication' (OT28). The impact of PSF was also described as 'holistic', with multiple categories ('physical and cognitive') or specific aspects of impact ('adversely effects patients sleep, appetite, motivation(sic)' (PT25)).

Furthermore, respondents described the manifestation of PSF as particularly pernicious. Several reported that 
the fatigue experienced by stroke survivors was disproportionate to the cerebrovascular accident that had occurred, saying, '(PSF) has the highest impact in the least neurologically/physically impaired stroke patients' (PT16). One respondent described patients as only experiencing PSF when 'they start trying to get back to everyday activities' (OT56), and several indicated that fatigue affected patients' ability to communicate with their family.

\section{A medical condition: legitimisation Medicalisation of symptoms}

Responses emphasised the medical nature, and thus legitimacy, of PSF as a condition. PSF was described as a 'condition' or 'symptom' of stroke, from which patients were 'suffering'. Some respondents explicitly differentiated PSF from tiredness, writing that PSF was 'completely different to 'normal' tiredness' (OT11) or 'not the same as being tired' (OT32, PT18). Similarly medicalising were efforts to quantify the fatigue, with respondents employing phrases such as 'it can be measured, monitored and energy conservation strategies applied' (OT22).

Scientific language was used to support these assertions; participants attributed PSF to processes of damage and healing in the brain. Neurological explanations given for PSF included, 'the brain reorganising connections' (OT24), '(PSF happens) as neuroplasticity occurs' (PT26), and PSF being 'caused by the effect of stroke on hormones, neurotransmitters and cognitive load' (OT28). One respondent attributed fatigue 'in-part to impairment of the regulatory systems in the brain-disruption to the network connections' (OT54).

\section{Applying a framework}

In the absence of an existing formal classification framework, respondents offered a variety of systematic approaches to organise PSF into a number of constituent causes or effects. These included classifying fatigue into 'peripheral' vs 'central' and 'primary' vs 'secondary'. This is typified by the following response: 'there is usually a primary (ie, central or disease-specific) mechanism and a secondary (loss of fitness/function, mood/sleep related, etc) mechanism at work' (OT64).

\section{Different clinical approaches}

There was variation in understanding between therapists, which was both explicitly identified by respondents acknowledging differences and implicitly conveyed by variation between responses.

Several respondents highlighted the different approaches within their multidisciplinary teams (MDT). One therapist wrote, 'The doctors sometimes prescribe the patients modafinil ... patients are often reviewed by the psychologist and the fatigue can be identified but I think there is a universal lack of knowing the best way to treat these patients' (PT24). A community PT highlighted this difficulty more explicitly, writing, 'I do sometimes feel that OT \& PT can give slightly conflicting messages. I appreciate that pacing is important, but sometimes (phrasing) can be very vague and misinterpreted...I often explain this ...the OTs tend to be much more likely to encourage patients not to over do things (sic)... it can be a very tricky balancing act' (PT33). Another communitybased PT asserted that, '(There are) different approaches in each discipline. OT(s) have most knowledge on fatigue management. Nursing and psychiatrists have the least knowledge and skill set to (manage) fatigue' [PT48].

In addition to the acknowledgement of divergent opinions between health practitioners, there were substantial differences in the terminology used to define PSF. Respondents presented definitions that directly contradicted their peers. Key points of difference were whether PSF was termed 'fatigue' or 'tiredness' in responses, whether fatigue was related to activity undertaken, and what its prognosis was.

\section{Tiredness or fatigue?}

The majority of respondents distinguished between the concept of 'tiredness' and fatigue' or defined fatigue as a particularly intense variant of tiredness ('tiredness like no other'), as previously discussed. That these comments typically occurred at the start of the response suggests that this distinction was considered important. Other respondents used the terms 'tiredness' and 'fatigue' interchangeably, for example, 'A tiredness that is not replenished by sleep and ... can be physical, mental and emotional fatigue' (OT63). In other responses, it was unclear whether the terms were used synonymously ('feelings of mild to extreme tiredness' (PT32)). Some respondents appeared to define fatigue by its speed of onset, defining fatigue as, 'feeling very easily tired' (OT09) and '(to) get tired very quickly' [PT54].

\section{Role of activity?}

Though there was consensus that fatigue had a serious effect on patients' abilities to carry out activities of daily living (ADL), there were inconsistencies in relating fatigue to activity. Some respondents wrote that PSF could 'not be attributed to activity' (OT03) and was 'not related to the level of activity a person undertakes' (OT20). One felt PSF was 'characterised by not being associated with recent levels of activity' (OT31). Conversely, others felt that the key to managing fatigue was to "understand ... how activity affects these (energy) levels' (OT22), and use 'pacing' as a strategy. Some responses were more equivocal, writing 'impact is not necessarily related to activity levels' (PT16) or 'not always linked to activity completed' (OT32). Others indicated that fatigue was disproportionate to activity.

Respondents consistently referred to rest when defining PSF. Therapists felt rest or a lack thereof affected patients' experience of PSF and should be considered when assessing for clinically significant fatigue. One stated 'the best thing is to encourage the person to take complete rest ... before doing anything else' (OT12), while others agreed; the fatigue 'reduces over time if adequate rest (is) taken (PT63)' and that it 'requires frequent rest periods' 
(OT23). Others mentioned rest but conversely were of the opinion that the condition 'does not necessarily resolve following rest' (OT06) and is 'not eased by rest' (OT31). Others framed it as more of a diagnostic symptom, stating that PSF is 'an absolute need to sleep and rest' (PT55).

\section{Timeframe?}

Finally, there was a marked discrepancy between responses offering a timescale for PSF. Respondents suggested that PSF, 'lasts ... from weeks, to months to years or permanent' (PT06), 'usually improves 1-2 years post-stroke' (PT53), and 'may (last) for several months or even years' (OT56). Similar in style were the responses, 'inability to participate in physio sessions longer than 15-20 min' (PT55) and 'usually improves 1-2 years poststroke' (PT53). Other responses addressed progression more vaguely, describing PSF as 'ongoing', or lessening 'with time'.

\section{Differences by professional background}

We did not discern differences between how OT and PT communicated their understanding of PSF, though PT used medicalised terminology such as 'symptom' and 'condition' more frequently than OT. Length of clinical experience also appeared to have little impact on responses from therapists with between 5 and 20 years specialisation in stroke. Therapists with over 20 years' experience alluded more frequently to the effect of PSF on ADLs and patient energy levels, often providing shorter and less holistic responses. Individuals with up to 5 years' experience in the specialism demonstrated a notably medicalised approach.

We found that respondents in the acute setting typically used 'medicalised' terminology compared with those working in the community, among whom metaphor and patient expression were more common. Among the 25 respondents who identified themselves as working in acute care, answers were characterised by the medicalisation of PSF, and by the conceptualisation of fatigue as a symptom of vascular injury. There was an emphasis on the frequency of occurrence of PSF. Respondents were more likely to use statistics for emphasis. Respondents who identified themselves as working in acute care tended to use terminology more similar to a 'dictionary definition' of the condition. Conversely, those working in subacute (24) or primary care (3) tended to define stroke in terms of its lived impact, particularly with regard to rehabilitation and the effect of PSF on specific tasks, effort and rest. Subacute and primary care-based respondents were more likely to describe PSF in terms of impact on daily life and patient engagement with rehabilitation sessions, or in a holistic manner.

The majority of respondents identified themselves as working in a community setting (85). These responses were characterised by a focus on the clinical management, lived experience of PSF, and the use of metaphor. Though metaphor was a common feature of responses, it was particularly characteristic among community-based therapists. Several community-based respondents relayed patient descriptors of fatigue as part of their definition, such as "trying to "think through treacle" (OT18). Almost all respondents who commented on variation in definitions and limitations to a common understanding of PSF, identified themselves as a community therapist. Though responses from this group displayed the most variation in definition of the key features of PSF, this may simply reflect the larger number of respondents from this setting.

\section{DISCUSSION}

\section{Principal findings}

Respondents portrayed PSF as a highly debilitating condition which deserved greater attention. Medicalisation was viewed positively, as it reduced the stigma associated with this 'invisible' condition. Metaphor was used to convey the serious and debilitating nature of PSF. There was a lack of consistency regarding key features of the condition, in particular over terminology use (eg, 'tiredness' or 'fatigue'), treatment (eg, the role of exercise) and prognosis. We found that respondents in the acute setting typically used 'medicalised' terminology compared with those working in the community, among whom metaphor and patient expression were more common.

\section{Comparison to relevant literature}

An overview of the fatigue literature

In 1971, McFarland stated that 'fatigue' is one of the most used and yet most poorly understood words in the English language. ${ }^{21}$ Despite a sharp increase in published literature on fatigue over the last 30 years, and on PSF specifically in the past two decades, this appears to still hold true. When compared with other post-stroke and chronic conditions, research on PSF is still in its infancy; little research has targeted fatigue after stroke as the primary outcome, particularly regarding management and clinical understanding. Mechanistic work is ongoing, which will further strengthen therapist and patient understanding. ${ }^{141522}$ However, due to a lack of definitive answers, it is unsurprising that our respondents felt there was insufficient research addressing PSF.

\section{The role of stroke survivor perspective}

Though several qualitative studies have focused on stroke survivors' understanding of PSF, previous literature has not examined healthcare professional understanding. Throughout the survey responses, metaphor and simple descriptive language were commonly used to emphasise the impact of PSF on stroke survivors, reflecting a central position of patient experience in clinical thinking around PSF. Several of the core characteristics identified by Eilertsen $e t a l$ s model of stroke survivors' experiences of $\mathrm{PSF}^{23}$ resonate with how therapists understood and defined the condition in our study. In both studies, individuals described PSF as an 'invisible disability' associated with stigmatisation, related fatigue to an 'abnormal' need to rest, and highlighted the lack of understanding and 
definition regarding PSF. Indeed, the authors felt that the 'medicalisation' identified in this dataset was employed to combat the evident stigma of PSF.

\section{Inclusion in clinical guidelines}

Collectively, the responses emphasised the importance of addressing PSF as a key post-stroke morbidity, and doing so in a holistic manner. Inclusion in clinical guidelines might improve recognition and guide practice. However, it is only recently starting to be included within prominent clinical guidelines. ${ }^{192425}$ As Eilertsen and colleagues argue, this lack of coverage may reflect the relative lack of high quality research in the field. ${ }^{23}$ In order to legitimise PSF, therapists emphasise its medical nature, debilitative characteristics and direct causative relationship with stroke. As Young et al have demonstrated, medical language increases perceived severity and condition representativeness, especially for less well-known conditions. ${ }^{2627}$

\section{Legitimacy of PSF in the multidisciplinary team}

The extent to which therapists legitimise a patient's symptoms can play a significant role in how the individual understands and navigates their condition. This is reflected often in fatigue literature; patients with rheumatoid arthritis report fatigue as overwhelming, uncontrollable and ignored, ${ }^{28}$ and studies indicate that it rarely forms a treatment target. By contrast, a survey of cancerspecialist therapists found that all professional groups overestimated the effects of fatigue compared with patient reports, ${ }^{29}$ though this may have been affected by the low response rate and degree of specialisation of the respondents involved. How far therapists engage with PSF may vary within a team. It is interesting that the open ended question we used led to discussion of the tensions involved in managing PSF within an MDT, indicating that this may be a particularly pertinent issue.

\section{Strengths and weaknesses}

The use of an online survey allowed respondents to participate from far reaching geographical locations and removes the influence of an interviewer on responses. Nevertheless, the open nature of the survey question may have resulted in different interpretations of the study question, and our descriptive data is hindered by the inability to question respondents further. ${ }^{29}$ Though the survey was targeted to specialised therapists and responses were submitted by a diverse demographic of healthcare practitioners, the response rate was low, at $8 \%$. This could mean that responses were taken primarily by healthcare professionals with greater interest and knowledge of PSF. Conversely, we had a relatively large number of respondents for a qualitative study, so it is unlikely that we failed to capture important themes. Although PTs and OTs often lead fatigue management programmes, other members of the MDT were not invited to take part which may limit the generalisability of our findings to other healthcare professionals. Qualitative analysis enabled the comprehensive and in-depth analysis of each response, however this precludes any broader generalisations about clinical practice as a whole.

\section{Clinical implications}

PSF has been reported as an unmet need that is often misunderstood. ${ }^{19}$ Our findings suggest that part of the problem may be that therapists working with stroke survivors conceptualise, explain and manage PSF in different ways. This finding helps explain some of the findings of qualitative studies carried out with stroke survivors and care givers. ${ }^{25}$ By drawing on both perspectives, guidance could be developed to support therapists assessing and educating their patients on PSF at all stages of the clinical pathway. This task would be made easier by a strong evidence-based framework for describing and explaining PSF in clinical practice and if there was a single widely adopted definition of PSF. Research into causes and management of PSF remains a priority.

\section{Twitter Karen Thomas @Karen_ThPCU and Clarissa Hjalmarsson @clarryhj}

Acknowledgements We are grateful to all survey respondents who took the time to participate in our research. Many thanks to Donna Malley for her guidance and clinical expertise at the inception of this project.

Contributors $\mathrm{KT}$ and RM contributed to the initial design of the study. $\mathrm{KT}$ and $\mathrm{CH}$ conducted data analysis and theme creation. $\mathrm{KT}, \mathrm{JM}, \mathrm{CH}$ and $\mathrm{RM}$ contributed to manuscript writing and editing.

Funding This study was funded by the Malati Kanbur Studentship fund kindly donated by Ravi Kanbur and Margaret Grieco to Lucy Cavendish College, constituent college of the University of Cambridge. JM is supported by an NIHR Senior Investigator award.

Competing interests None declared.

Patient consent for publication Not required.

Ethics approval All documentation was approved by Cambridge University Psychology Ethics Committee (PRE.2017.092).

Provenance and peer review Not commissioned; externally peer reviewed.

Data availability statement No data are available.

Open access This is an open access article distributed in accordance with the Creative Commons Attribution Non Commercial (CC BY-NC 4.0) license, which permits others to distribute, remix, adapt, build upon this work non-commercially, and license their derivative works on different terms, provided the original work is properly cited, appropriate credit is given, any changes made indicated, and the use is non-commercial. See: http://creativecommons.org/licenses/by-nc/4.0/.

\section{ORCID iDs}

Karen Thomas http://orcid.org/0000-0001-9360-0862

Clarissa Hjalmarsson http://orcid.org/0000-0003-2961-5984

\section{REFERENCES}

1 McKevitt C, Fudge N, Redfern J, et al. Self-Reported long-term needs after stroke. Stroke 2011;42:1398-403.

2 Cumming TB, Packer M, Kramer SF, et al. The prevalence of fatigue after stroke: a systematic review and meta-analysis. Int J Stroke 2016;11:968-77.

3 Glader E-L, Stegmayr B, Asplund K. Poststroke fatigue: a 2year follow-up study of stroke patients in Sweden. Stroke 2002;33:1327-33.

4 De Groot MH, Phillips SJ, Eskes GA. Fatigue associated with stroke and other neurologic conditions: implications for stroke rehabilitation. Arch Phys Med Rehabil 2003;84:1714-20.

5 Naess $\mathrm{H}$, Lunde L, Brogger J, et al. Fatigue among stroke patients on long-term follow-up. The Bergen stroke study. J Neurol Sci 2012;312:138-41. 
6 Mead G, Lynch J, Greig C, et al. Evaluation of fatigue scales in stroke patients. Stroke 2007:38:2090-5.

7 Wu S, Kutlubaev MA, Chun H-YY, et al. Interventions for post-stroke fatigue. Cochrane Database Syst Rev 2015;22. Art No: CD007030.

8 Annoni J-M, Staub F, Bogousslavsky J, et al. Frequency, characterisation and therapies of fatigue after stroke. Neurological Sciences 2008;29:244-6.

9 Thomas K, Gamlin C, De Simoni A, et al. How is poststroke fatigue understood by stroke survivors and carers? A thematic analysis of an online discussion forum. BMJ Open 2019;9:e028958.

10 Acciarresi M, Bogousslavsky J, Paciaroni M. Post-Stroke fatigue: epidemiology, clinical characteristics and treatment. Eur Neurol 2014;72:255-61.

11 Kuppuswamy A, Rothwell J, Ward N. A model of poststroke fatigue based on sensorimotor deficits. Curr Opin Neurol 2015;28:582-6.

12 Giulio CD, Daniele F, Tipton CM. Angelo Mosso and muscular fatigue: 116 years after the first Congress of physiologists: IUPS commemoration. Adv Physiol Educ 2006;30:51-7.

13 De Doncker W, Dantzer R, Ormstad H, et al. Mechanisms of poststroke fatigue. J Neurol Neurosurg Psychiatry 2018;89:287-93.

14 Kuppuswamy A. The fatigue conundrum. Brain 2017;140:2240-5.

15 Stephan KE, Manjaly ZM, Mathys CD, et al. Allostatic self-efficacy: a metacognitive theory of Dyshomeostasis-Induced fatigue and depression. Front Hum Neurosci 2016;10.

16 Lynch J, Mead G, Greig C, et al. Fatigue after stroke: the development and evaluation of a case definition. J Psychosom Res 2007;63:539-44.

17 Pollock A, St George B, Fenton M, et al. Top ten research priorities relating to life after stroke. Lancet Neurol 2012;11.

18 Hinkle JL, Becker KJ, Kim JS, et al. Poststroke fatigue: emerging evidence and approaches to management: a scientific statement for healthcare professionals from the American Heart Association. Stroke 2017;48:e159-70.
19 O'Brien BC, Harris IB, Beckman TJ, et al. Standards for reporting qualitative research: a synthesis of recommendations. Acad Med 2014;89:1245-51.

20 McFarland RA. Fatigue in industry. understanding fatigue in modern life. Ergonomics 1971;14:1-10.

21 Chaudhuri A, Behan PO. Fatigue in neurological disorders. Lancet 2004;363:978-88.

22 Eilertsen G, Ormstad H, Kirkevold M. Experiences of poststroke fatique: qualitative meta-synthesis. J Adv Nurs 2013;69:514-25.

23 National Institute for Health and Care Excellence. Stroke rehabilitation: long-term rehabilitation after stroke (clinical guideline CG162_. Secondary stroke rehabilitation: long-term rehabilitation after stroke (clinical guideline CG162), 2013. Available: http:/ guidance.nice.org.uk/CG162

24 Royal College of Physicians. National clinical guideline for stroke: fourth edition, 2012

25 Young M, Brooks L, Norman G. Found in translation: the impact of familiar symptom descriptions on diagnosis in novices. Med Educ 2007:41:1146-51.

26 Young ME, Norman GR, Humphreys KR. The role of medical language in changing public perceptions of illness. PLoS One 2008;3:e3875.

27 Hewlett S, Cockshott Z, Byron M, et al. Patients' perceptions of fatigue in rheumatoid arthritis: overwhelming, uncontrollable, ignored. Arthritis Rheum 2005;53:697-702.

28 Stone P, Ream E, Richardson A, et al. Cancer-related fatigue - a difference of opinion? Results of a multicentre survey of healthcare professionals, patients and caregivers. Eur J Cancer Care 2003;12:20-7.

29 Kelley K, Clark B, Brown V, et al. Good practice in the conduct and reporting of survey research. Int J Qual Health Care 2003;15:261-6. 